\title{
On the Error Term for the Number of Integral Ideals in Galois Extensions
}

\author{
Sanying Shi* \\ School of Mathematics, Hefei University of Technology, Hefei, China \\ *Corresponding author: vera123_99@hotmail.com \\ Received November 05, 2014; Revised December 05, 2014; Accepted December 18, 2014

\begin{abstract}
Suppose that $\mathrm{E}$ is an algebraic number field over the rational field $\mathbb{Q}$. Let $\mathrm{a}(\mathrm{n})$ be the number of integral ideals in $\mathrm{E}$ with norm $\mathrm{n}$ and $\Delta(\mathrm{x})$ denote the remainder term in the asymptotic formula of the 1-th integral power sum of $\mathrm{a}(\mathrm{n})$. In this paper the bound of the average behavior of $\Delta(\mathrm{x})$ is given. This result constitutes an improvement upon that of Lü and Wang for the error terms in mean value.
\end{abstract}

Keywords: dedekind zeta-function, dirichlet series, mean value

Cite This Article: Sanying Shi, "On the Error Term for the Number of Integral Ideals in Galois Extensions." Turkish Journal of Analysis and Number Theory, vol. 2, no. 6 (2014): 230-232. doi: 10.12691/tjant-2-6-8.

\section{Introduction and the Result}

Let $E$ be an algebraic number field of degree $d$ over the rational field $\mathbb{Q}$, and $\zeta(s, E)$ be its Dedekind zetafunction. Thus

$$
\zeta(s, E)=\sum_{\mathfrak{a}} \frac{1}{(N \mathfrak{a})^{s}}, \quad(\mathfrak{R e}(s)>1),
$$

where $\mathfrak{a}$ runs over all integral ideals of the field $E$, and $N \mathfrak{a}$ is the norm of $\mathfrak{a}$. If $a(n)$ denotes the number of integral ideals in $E$ with norm $n$, then we have

$$
\zeta(s, E)=\sum_{n=1}^{\infty} \frac{a(n)}{n^{s}}, \quad s=\sigma+i t, \quad \sigma>1 .
$$

It is known that $a(n)$ is a multiplicative function and satisfies

$$
a(n) \ll \tau(n)^{d},
$$

where $\tau(n)$ is the divisor function.

It is an important problem to study the function $a(n)$. In 1927, Landau [7] first proved that

$$
\sum_{n \leq x} a(n)=\alpha x+O\left(x^{1-\frac{2}{d+1}+\epsilon}\right)
$$

for any arbitrary algebraic number field of degree $d \geq 2$, where $\alpha$ is the residue of $\zeta(s, E)$ at its simple pole $s=1$.

It is hard to refine Landau's result. Later, Huxley and Watt [3] and Müller [9] improved the results for the quadratic and cubic fields, respectively.

Until 1993, Nowak [10] obtained the best result

$$
\sum_{n \leq x} a(n)=\alpha x+\left\{\begin{array}{l}
O\left(x^{\left.1-\frac{2}{d}+\frac{8}{d(5 d+2)}(\log x)^{\frac{10}{5 d+2}}\right),}, \text { for } 3 \leq d \leq 6\right. \\
O\left(x^{1-\frac{2}{d}+\frac{3}{2 d^{2}}(\log x)^{\frac{2}{d}}}\right), \quad \text { for } d \geq 7
\end{array}\right.
$$

for any arbitrary algebraic number field of degree $d \geq 3$.

In [1], Chandraseknaran and Good studied the $l$-th integral power sum of $a(n)$ in some Galois fields, and they showed that

Theorem 1.0. If $E$ is a Galois extension of $\mathbb{Q}$ of degree $d$, then for any $\epsilon>0$ and any integer $d \geq 2$, we have

$$
\sum_{n \leq x} a(n)^{l}=x Q_{m}(\log x)+O\left(x^{1-\frac{2}{m d}+\epsilon}\right)
$$

where $m=d^{l-1}$, and $Q_{l}(t)$ is a suitable polynomial in $t$ of degree $m-1$.

Recently, Lü and Wang [8] improved the classical result of [1] by replacing $\frac{2}{m d}$ with $\frac{3}{m d+6}$.

Motivated by $[2,4,5]$, the purpose of this paper is to study the remainder term in mean square, and we shall prove the following result.

Theorem 1.1 Subject to assumptions in Theorem 1.0, and define

$$
\Delta(x):=\sum_{n \leq x} a(n)^{l}-x Q_{m}(\log x) .
$$

Then we have

$$
\int_{1}^{X} \Delta^{2}(x) d x \ll_{\epsilon} X^{3-\frac{6}{m d+3}+\epsilon}
$$


for any given $\epsilon>0$.

Notations. As usual, the Vinogradov symbol $A \ll B$ means that $B$ is positive and the ratio $A / B$ is bounded. The letter $\epsilon$ denotes an arbitrary small positive number, not the same at each occurrence.

\section{Proof of Theorem 1.1}

To prove our Theorem, we need the following lemmas.

Lemma 2.1 Let $E / \mathbb{Q}$ be a Galois extension of degree $d$, and $a(n)$ be defined in (1). Define

$$
N_{l}(s)=\sum_{n=1}^{\infty} \frac{a(n)^{l}}{n^{s}},(\mathfrak{R e} s>1) .
$$

Then we have

$$
N_{l}(s)=\zeta^{m}(s, E) \cdot A_{1}(s)
$$

for any integer $l \geq 1$, where $m=d^{l-1}$, and $A_{1}(s)$ denotes a Dirichlet series, which is absolutely and uniformly convergent for $\mathfrak{R e}(s)>1 / 2$.

Proof. This is Lemma 2.1 in [8].

Lemma 2.2. Let $E$ be an algebraic number field of degree $d$, then

$$
\zeta(\sigma+i t, E) \ll(1+|t|)^{\frac{d}{3}(1-\sigma)+\epsilon},
$$

for $\frac{1}{2} \leq \sigma \leq 1+\epsilon$ and any fixed $\epsilon>0$.

Proof. By Lemma 2.2 in [8] and the PhragmenLindelöf principle for a strip (see, e.g. Theorem 5.53 in [6]), Lemma 2.2 follows immediately.

Now we begin to prove our theorem.

Let $E$ be a Galois extension of $\mathbb{Q}$ of degree $d$.

Recall $a(n)$ denotes the number of integral ideals in $E$ with norm $n$, and

$$
\zeta(s, E)=\sum_{n=1}^{\infty} \frac{a(n)}{n^{s}}, \quad s=\sigma+i t, \quad \sigma>1 .
$$

Let

$$
T=X^{\frac{3}{d^{l}+3}}
$$

From (1), (3) and Perron's formula (see Proposition 5.54 in [6], we get

$$
\sum_{n \leq x} a(n)^{l}=\frac{1}{2 \pi i} \int_{1+\epsilon-i T}^{1+\epsilon+i T} N_{l}(s) \frac{x^{s}}{s} d s+O\left(\frac{x^{1+\epsilon}}{T}\right) .
$$

By the property $N_{l}(s)$ only has a simple pole at $s=1$ for $\mathfrak{R e}(s)>\frac{1}{2}$ and Cauchy's residue theorem, we have

$$
\begin{gathered}
\sum_{n \leq x} a\left(n^{2}\right)^{l}=\frac{1}{2 \pi i}\left\{\int_{\frac{1}{2}+\epsilon-i T}^{\frac{1}{2}+\epsilon+i T}+\int_{\frac{1}{2}+\epsilon+i T}^{1+\epsilon+i T}+\int_{1+\epsilon-i T}^{\frac{1}{2}+\epsilon-i T}\right\} N_{l}(s) \frac{x^{s}}{s} d s \\
+\underset{s=1}{\operatorname{Res}} N_{l}(s) x+O\left(\frac{x^{1+\epsilon}}{T}\right) \\
=x Q_{m}(\log x)+J_{1}(x)+J_{2}(x)+J_{3}(x)+O\left(x^{1+\epsilon} T^{-1}\right) .
\end{gathered}
$$

where $m=d^{l-1}$, and $Q_{m}(t)$ is a suitable polynomial in $t$ of degree $m-1$.

From the definition of $\Delta(x)$ in $(2)$, we have

$$
\Delta(x)=J_{1}(x)+J_{2}(x)+J_{3}(x)+O\left(x^{1+\epsilon} T^{-1}\right) .
$$

Therefore to prove Theorem 1.1, we shall prove the following results.

$$
\int_{1}^{X} J_{i}^{2}(x) d x \ll_{\epsilon} X^{3-\frac{6}{m d+3}+\epsilon}, i=1,2,3
$$

and

$$
\int_{1}^{X}\left(O\left(\frac{x^{1+\epsilon}}{T}\right)\right)^{2} d x \ll_{\epsilon} X^{3-\frac{6}{m d+3}+\epsilon}
$$

It is easy to get

$$
\int_{1}^{X}\left(O\left(\frac{x^{1+\epsilon}}{T}\right)\right)^{2} d x \ll \frac{X^{3+\epsilon}}{T^{2}} \ll X^{3-\frac{6}{m d+3}+\epsilon} .
$$

Now we consider the integral $J_{1}(x)$. We have

$$
J_{1}(x)=\frac{1}{2 \pi} \int_{-T}^{T} N_{l}\left(\frac{1}{2}+\epsilon+i t\right) \frac{x^{\frac{1}{2}+\epsilon+i t}}{\frac{1}{2}+\epsilon+i t} d t .
$$

Then

$$
\int_{1}^{X} J_{1}^{2}(x) d x=\frac{1}{4 \pi^{2}} \int_{1}^{X}\left(\int_{-T}^{T} N_{l}\left(\frac{1}{2}+\epsilon+i t_{1}\right) \frac{x^{\frac{1}{2}+\epsilon+i t_{1}}}{\frac{1}{2}+\epsilon+i t_{1}} d t_{1}\right.
$$$$
\left.\times \int_{-T}^{T} \overline{N_{l}\left(\frac{1}{2}+\epsilon+i t_{2}\right)} \frac{x^{\frac{1}{2}+\epsilon-i t_{2}}}{\frac{1}{2}+\epsilon-i t_{2}} d t_{2}\right) d x
$$$$
=\frac{1}{4 \pi^{2}} \int_{-T}^{T} \int_{-T}^{T} \frac{N_{l}\left(\frac{1}{2}+\epsilon+i t_{1}\right) \overline{N_{l}\left(\frac{1}{2}+\epsilon+i t_{2}\right)}}{\left(\frac{1}{2}+\epsilon+i t_{1}\right)\left(\frac{1}{2}+\epsilon-i t_{2}\right)}
$$

$$
\times\left(\int_{1}^{X} x^{1+2 \epsilon+i\left(t_{1}-t_{2}\right)}\right) d x d t_{1} d t_{2}
$$

$\ll X^{2+2 \epsilon} \int_{-T}^{T} d t_{1} \int_{-T}^{T} \frac{\left|N_{l}\left(\frac{1}{2}+\epsilon+i t_{1}\right) \| N_{l}\left(\frac{1}{2}+\epsilon+i t_{2}\right)\right|}{\left(1+\left|t_{1}\right|\right)\left(1+\left|t_{2}\right|\right)\left(1+\left|t_{1}-t_{2}\right|\right)} d t_{2}$ 


$$
\begin{gathered}
\ll X^{2+2 \epsilon} \int_{-T}^{T} d t_{1} \int_{-T}^{T}\left(\frac{\left|N_{l}\left(\frac{1}{2}+\epsilon+i t_{1}\right)\right|^{2}}{\left(1+\left|t_{1}\right|\right)^{2}}\right. \\
\left.+\frac{\left|N_{l}\left(\frac{1}{2}+\epsilon+i t_{2}\right)\right|^{2}}{\left(1+\left|t_{2}\right|\right)^{2}}\right) \frac{d t_{2}}{1+\left|t_{1}-t_{2}\right|} \\
\ll X^{2+2 \epsilon} \int_{-T}^{T} \frac{\left|N_{l}\left(\frac{1}{2}+\epsilon+i t_{1}\right)\right|^{2}}{\left(1+\left|t_{1}\right|\right)^{2}} d t_{1} \int_{-T}^{T} \frac{d t_{2}}{1+\left|t_{1}-t_{2}\right|} .
\end{gathered}
$$

To go further, we get

$$
\begin{aligned}
\int_{-T}^{T} \frac{d t_{2}}{1+\left|t_{1}-t_{2}\right|} & \ll \int_{t_{1}-1}^{t_{1}+1} d t_{2}+\left(\int_{t_{1}+1}^{T}+\int_{-T}^{t_{1}-1}\right) \frac{d t_{2}}{\left|t_{1}-t_{2}\right|} \\
& \ll 1+\int_{t_{1}+1}^{T} \frac{d t_{2}}{\left|t_{1}-t_{2}\right|} \\
& \ll \int_{1}^{T+\left|t_{1}\right|} \frac{d t}{t} \ll \log 2 T .
\end{aligned}
$$

By (7) and (8)

$$
\int_{1}^{X} J_{1}^{2}(x) d x \ll X^{2+3 \epsilon} \int_{-T}^{T} \frac{\left|N_{l}\left(\frac{1}{2}+\epsilon+i t_{1}\right)\right|^{2}}{\left(1+\left|t_{1}\right|\right)^{2}} d t_{1} .
$$

From (9), Lemma 2.1 and 2.3, we have (for $d \geq 3$ )

$$
\begin{aligned}
\int_{1}^{X} J_{1}^{2}(x) d x & \ll X^{2+3 \epsilon}+X^{2+3 \epsilon} \int_{1}^{T} \mid \zeta^{d-1}\left(\frac{1}{2}+\epsilon+i t, E\right) \\
& \times\left. A_{1}\left(\frac{1}{2}+\epsilon+i t_{1}\right)\right|^{2} t^{-2} d t \\
& \ll X^{2+3 \epsilon}+X^{2+3 \epsilon} \int_{1}^{T}\left|\zeta^{m}\left(\frac{1}{2}+\epsilon+i t, E\right)\right|^{2} t^{-2} d t \\
& \ll X^{2+3 \epsilon}+X^{2+3 \epsilon} \int_{1}^{T}\left(\frac{m d}{6}+\epsilon\right)^{2} t^{-2} d t \\
& \ll X^{2+3 \epsilon}+X^{2+4 \epsilon} T^{\frac{m d}{3}-1} \\
& \ll X^{3-\frac{6}{m d+3}+\epsilon} .
\end{aligned}
$$

Finally we estimate trivial bounds of the integrals $J_{2}(x), J_{3}(x)$. By Lemma 2.2, we get

$$
\begin{aligned}
J_{2}(x)+J_{3}(x) & \ll \int_{\frac{1}{2}+\epsilon}^{1+\epsilon} x^{\sigma}\left|\zeta^{m}(\sigma+i T, E)\right| T^{-1} d \sigma \\
& \ll \max _{1 / 2+\epsilon \leq \sigma \leq 1+\epsilon} x^{\sigma} T^{\frac{m d}{3}(1-\sigma)+\epsilon} T^{-1} \\
& =\max _{\frac{1}{2}+\epsilon \leq \sigma \leq 1+\epsilon}\left(\frac{x}{T^{m d / 3}}\right)^{\sigma} T^{\frac{m d}{3}-1+\epsilon} \\
& \ll \frac{x^{1+\epsilon}}{T}+x^{\frac{1}{2}+\epsilon} T^{\frac{d^{l}}{6}-1+\epsilon},
\end{aligned}
$$

which yields

$$
\begin{aligned}
\int_{1}^{X}\left(J_{2}(x)+J_{3}(x)\right)^{2} d x & \ll \int_{1}^{X}\left(\frac{x^{1+\epsilon}}{T}+x^{\frac{1}{2}+\epsilon} T^{\frac{m d}{6}-1+\epsilon}\right)^{2} d x \\
& \ll \int_{1}^{X}\left(\frac{x^{1+\epsilon}}{T}\right)^{2} \\
& \ll \frac{X^{3+\epsilon}}{T^{2}}+X^{2+2 \epsilon} T^{\frac{m d}{3}-2+2 \epsilon} \\
& \ll X^{3-\frac{6}{m d+3}+\epsilon} .
\end{aligned}
$$

The inequalities (4), (5) immediately follow from (6), (10) and (11). That is,

$$
\int_{1}^{X} \Delta^{2}(x) d x \ll_{\epsilon} X^{3-\frac{6}{m d+3}+\epsilon} .
$$

Then this completes the proof of Theorem 1.1.

\section{Acknowledgement}

This work is supported by The National Science Foundation of China (grant no. 11201107 and 11071186) and by Natural Science Foundation of Anhui province (Grant No. 1208085QA01).

\section{References}

[1] Chandraseknaran K., Good A., On the number of integral ideals in Galois extensions, Monatsh. Math., 95. 99-109. 1983.

[2] Heath-Brown D. R., The number of Abelian groups of order at most $x$, Journtes Arithmttiques, Luminy 1989.

[3] Huxley M. N., Watt N., The number of ideals in a quadratic field II, Israel J. Math. Part A, 120, 125-153. 2000,

[4] Ivic A., The number of finite non-isomorphic Abelian groups in mean square, Hardy-Ramanujan J., 9, 17-23. 1986.

[5] Ivic A., On the Error Term for the Counting Functions of Finite Abelian Groups, Monatsh. Math. 114, 115-124. 1992.

[6] Iwaniec H., Kowalski E., Analytic Number Theory, Amer. Math. Soc. Colloq. Publ., 53, 204-216. 2004.

[7] Landau E., Einführung in die elementare and analytische Theorie der algebraischen Zahlen und der Ideale, Teubner, 1927.

[8] Lü G., Wang Y., Note on the number of integral ideals in Galois extension, Sci. China Ser. A, 53, 2417-2424. 2010.

[9] Müller W., On the distribution of ideals in cubic number fields, Monatsh. Math., 106, 211-219. 1988.

[10] Nowak W.G., On the distribution of integral ideals in algebraic number theory fields, Math. Nachr., 161, 59-74. 1993. 